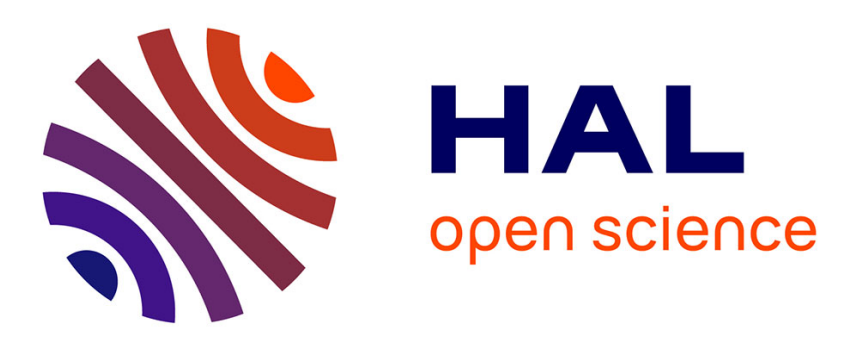

\title{
Stability of convection in a horizontal channel subjected to a longitudinal temperature gradient. Part 2. Effect of a magnetic field
}

D.V. Lyubimov, T.P. Lyubimova, A.B. Perminov, Daniel Henry, Hamda Ben Hadid

\section{To cite this version:}

D.V. Lyubimov, T.P. Lyubimova, A.B. Perminov, Daniel Henry, Hamda Ben Hadid. Stability of convection in a horizontal channel subjected to a longitudinal temperature gradient. Part 2. Effect of a magnetic field. Journal of Fluid Mechanics, 2009, 635, pp.297-319. 10.1017/S0022112009007575 . hal-00441951

\section{HAL Id: hal-00441951 \\ https://hal.science/hal-00441951}

Submitted on 7 Jul 2010

HAL is a multi-disciplinary open access archive for the deposit and dissemination of scientific research documents, whether they are published or not. The documents may come from teaching and research institutions in France or abroad, or from public or private research centers.
L'archive ouverte pluridisciplinaire HAL, est destinée au dépôt et à la diffusion de documents scientifiques de niveau recherche, publiés ou non, émanant des établissements d'enseignement et de recherche français ou étrangers, des laboratoires publics ou privés. 


\title{
Stability of convection in a horizontal channel subjected to a longitudinal temperature gradient. Part 2. Effect of a magnetic field
}

\author{
D. V. LYUBIMOV ${ }^{1}$, T. P. LYUBI M OVA ${ }^{2}$, A. B. PERMINOV \\ D. HEN R Y ${ }^{4}$ AND H. BEN HADID ${ }^{4} \dagger$ \\ ${ }^{1}$ Perm State University, 15 Bukirev Street, 614990 Perm, Russia \\ ${ }^{2}$ Institute of Continuous Media Mechanics UB RAS, 1 Koroleva Street, 614013 Perm, Russia \\ ${ }^{3}$ Perm State Technical University, 29a Komsomolsky Prospect, 614000 Perm, Russia \\ ${ }^{4}$ Université de Lyon, École Centrale de Lyon/Université Lyon 1/INSA de Lyon, Laboratoire de \\ Mécanique des Fluides et d'Acoustique, UMR-CNRS 5509, 43 Bd du 11 Novembre 1918, \\ 69622 Villeurbanne Cedex, France
}

(Received 20 December 2007; revised 30 March 2009; accepted 9 April 2009)

The stabilization of buoyant flows by a magnetic field is an important matter for crystal growth applications. It is studied here when the cavity is an infinite channel with rectangular cross-section typical of horizontal Bridgman configurations and when the magnetic field is applied in the vertical and transverse directions. The steady basic flow solution is first calculated: the usual counter flow structure is modified by the magnetic field and evolves towards jets in the cross-section corners when the magnetic field is vertical and towards a more uniform structure in the transverse direction when the magnetic field is transverse. The stability results show a very good stabilization of the convective flows for a vertical magnetic field with exponential increases of the thresholds for any width of the channel and for various Prandtl numbers $P r$. The results for a transverse magnetic field are more surprising as a destabilizing effect corresponding to an initial decrease of the thresholds is obtained at $\operatorname{Pr}=0$ and for small channel widths. A kinetic energy budget at the thresholds reveals that the main destabilizing factor is connected to the vertical shear of the longitudinal basic flow and that it is the modifications affecting this shear energy which are mainly responsible for the variation of the thresholds when a magnetic field is applied.

\section{Introduction}

In crystal growth process from the melt, time-dependent oscillatory flow motion implies unavoidable crystal-liquid interface oscillations which influence solute segregation in the crystal. Thus, controlling the fluid motion to reduce or to eliminate the temperature fluctuations becomes necessary to improve the crystal quality. In material processing, static or rotating magnetic fields are used for a contactless control of fluid flow and mass transport during the processes. Both static and rotating magnetic field can lead to a suppression of the temperature fluctuations (Hurle, Jakeman \& Johnson 1974; Dold \& Benz 1995, 1997; Davoust et al. 1999; Juel et al. 1999). However, the two fields act on the fluid in different ways: for a

$\dagger$ Email address for correspondence: hamda.benhadid@univ-lyon1.fr 
static field the strength of the Lorentz forces (body forces) depends on the vigour of the convective flow and diminishes as the intensity of the convective flow decreases, whereas rotating magnetic field can induce a flow even in a melt initially at rest.

In the last decades, the use of magnetic fields in material processing was mainly limited to stationary magnetic fields owing to their damping effects on the melt motion and thus the reduction of temperature fluctuations. The strength of the applied stationary field is generally in the range of several hundred millitesla (Series \& Hurle 1991; Hurle 1993). The pioneering experimental works on this problem were carried out by Utech \& Flemings (1966) and Hurle et al. (1974). They observed thermal oscillations in the flow and showed that a magnetic field applied orthogonally to the main convective flow can be used to damp the oscillations. Juel et al. (1999) investigated the effect of horizontal transverse magnetic field on temperature fluctuations in a horizontal rectangular cavity of aspect ratio four. Their combined experimental and numerical investigations show that the basic flow state in the rectangular box filled with liquid gallium is modified into a new two-dimensional configuration. A value of $H a=64$ was sufficient to damp the initial temperature fluctuations caused by buoyancy-driven convection. Based on the vertical temperature difference in the centre of the cavity, the comparisons between the experimental and numerical results are very good. In both cases, the vertical temperature difference and similarly the maximum horizontal and vertical velocities have been found to vary approximately as $H a^{-1}$ for $H a>100$. Experiments by Davoust et al. (1999) demonstrated the damping of thermogravitational flow of mercury in a horizontal circular cylindrical cavity of aspect ratio 10 by means of a uniform vertical magnetic field. The structure of the steady flow is investigated at large values of Hartmann number up to 235 and a good qualitative agreement is found with the asymptotic predictions of Garandet, Alboussière \& Moreau (1992). A study of time-dependent convective flow is also performed which shows in particular that damping is found to occur for small values of the Hartmann number between 1 and 10 . Recent experiments by Hof, Juel \& Mullin (2003, 2005) focused on the directional effect of the magnetic field in a rectangular enclosure of relative dimensions $5 \times 1.3 \times 1$ (length $\times$ width $\times$ height). They studied the damping of the steady convective flow (Hof et al. 2003) and the stabilization of the oscillatory transitions (Hof et al. 2005). They found that the oscillatory thresholds scale exponentially with $\mathrm{Ha}$ for the three principal orientations of the magnetic field. For both steady and oscillatory flows, the magnetic field is most effective when it is applied in the vertical direction, compared with the transverse and longitudinal directions.

The control of electrically conducting fluid flows with the help of external magnetic fields seems to be promising. Therefore, current research has intensified in the field of magnetohydrodynamic convection and several new results were obtained in the last decade. In this context, we particularly refer to the papers of Garandet et al. (1992) and Alboussière, Garandet \& Moreau (1993, 1996). These authors proposed analytical derivations often based on asymptotic models at large Hartmann number. For a vertical magnetic field, Garandet et al. (1992) first considered a two-dimensional rectangular cavity and showed that the decrease of the parallel flow in the bulk varied as $\mathrm{Ha}^{-2}$. The influence of the cross-section shape on the magnetic damping in the case of long horizontal cavities was then studied analytically in Alboussière et al. (1993). In the case of electrically insulating boundaries, the nature of the symmetry of the cross-section was found to govern the magnitude and the structure of the damped velocity. Indeed, the magnetically damped convection velocity varies as $\mathrm{Ha}^{-2}$ when the cross-section has a horizontal plane of symmetry, and $\mathrm{Ha}^{-1}$ for non-symmetrical 
shapes. In contrary, when the walls are perfectly conducting, the damped velocity always varies as $\mathrm{Ha}^{-2}$. Finally, Alboussière et al. (1996) considered magnetic fields of any orientation and obtained interesting results on the damping of the flows which depends on the symmetry of the configuration described as regular or singular.

Ozoe \& Okada (1989) reported the results of a three-dimensional numerical investigation of the directional effect of a steady magnetic field on side wall convection in a cubic cavity filled with molten silicon. They found that the longitudinal orientation of the magnetic field was most effective for suppressing convection, whereas the transverse direction was least effective. These results are questionable as the mesh used for the calculations was probably too coarse to get very accurate results. Nevertheless, the results of an experimental study using gallium in a similar cavity are later reported by the same authors (Okada \& Ozoe 1992), which qualitatively confirm the numerical findings with a larger decrease of the Nusselt number for the longitudinal and vertical magnetic fields. Ben Hadid \& Henry $(1996,1997)$ carried out calculations of three-dimensional flows of mercury for $\mathrm{Pr}=0.026$ in both cylindrical and rectangular cavities of aspect ratio 4, for different orientations of the magnetic field. They found good agreements with the analytical estimates of Garandet et al. (1992) and Alboussière et al. (1993, 1996) for the damping of the velocity field. For the rectangular cavity, the investigations include free-surface effects. Interesting changes in the flow structure are reported and these appear to be closely linked to the distribution of the induced electric currents and their interaction with the applied magnetic field. Three-dimensional numerical simulations of melt convection carried out by Baumgartl \& Müller (1992) in a cylindrical geometry submitted to a constant magnetic field show that only the models including the electric potential equation agree well with the experimental results. The numerical results of Baumgartl, Hubert \& Müller (1993) show that for $H a \sim 20(|B| \sim 7 m T)$ flow fluctuations with typical amplitudes of about $4 \%$ of the mean values are suppressed and the resulting steady flow has essentially the same features as the unsteady one. Ben Hadid, Henry \& Touihri (1997b) confirmed that a static field of small intensity can be used to significantly damp temperature fluctuations caused by time-dependent buoyancydriven convection. More recently, Henry et al. $(2008 a, b)$ studied the effect of the magnetic field on the instability thresholds in parallelepipedic cavities. In a $4 \times 2 \times 1$ cavity, they obtained exponential increases of the thresholds with the intensity of the magnetic field and showed that the vertical direction of the magnetic field was most effective, confirming the experimental results of Hof et al. (2005). They further proposed a kinetic energy analysis where they showed that the evolution of the threshold was strongly connected to changes affecting the main destabilizing shear energy term.

From these studies, it can be generally stated that an increase in the strength of the applied magnetic field leads to several fundamental changes in the properties of thermal convection. The convective circulation progressively loses its intensity and when $\mathrm{Ha}$ reaches a certain critical value, which is found to depend on the direction of the applied magnetic field (longitudinal, vertical or transverse), decrease of the flow intensity takes on an asymptotic form with important changes in the structure of the flow circulation. For high Hartman number, the flow structure exhibits three separated regions: the core flow, the Hartman layers of thickness $\mathrm{Ha}^{-1}$ which develop along walls which are perpendicular to the applied magnetic field and the parallel layers of thickness $\mathrm{Ha}^{-1 / 2}$ appearing along walls parallel to the applied magnetic field. Note that viscous effects are confined to these layers, whereas the remainder of the flow, namely the core flow, may be considered as inviscid. These modifications 


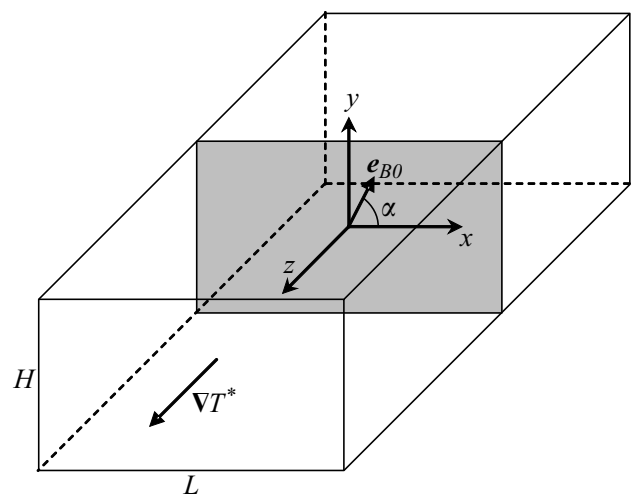

Figure 1. Studied configuration.

at high Hartmann number are mainly governed by the electric current circulation and can be explained by analysing its paths and the resulting Lorentz forces. In the case of electrically insulating boundaries, for instance, the resulting electric currents form closed loops inside the cavity, and a significant braking effect on the convection is generated in regions where the electric current vectors are perpendicular to the applied magnetic field, whereas a weak braking effect is obtained in regions where the electric current is rather parallel to the applied magnetic field, generally near side walls where over velocities will develop.

In this paper the influence of a static magnetic field on the stability of buoyancy driven convection is investigated. As in Lyubimova et al. (2008), we consider an infinitely long horizontal channel of rectangular cross-section in which the convective flow is generated by a longitudinal temperature gradient. This flow is submitted to a static magnetic field perpendicular to the channel axis, either vertical or horizontal. We present results on the influence of the magnetic field on the convective flows and on the instabilities which develop in such flows, for various cross-sections and in a wide range of Prandtl number values. A kinetic energy analysis is finally performed to shed light on the physical mechanisms involved in the observed variations of the thresholds.

\section{Governing equations and boundary conditions}

A viscous non-compressible fluid with constant electrical conductivity $\sigma_{e}$ fills a horizontal channel of rectangular cross-section $S$ of height $H$ (along the $y$-axis) and width $L$ (along the $x$-axis) (figure 1 ). An aspect ratio is defined for the channel by $l=L / H$. The fluid is subject to a temperature gradient $(\nabla T)^{*}$ which is colinear with the $z$-axis (the third axis of the cavity) and to a constant magnetic field $\vec{B}_{0}$. The fluid is assumed to be Newtonian with constant kinematic viscosity $v$ and thermal diffusivity $\kappa$. The usual Boussinesq approximation is employed, which means that the density variations are restricted to the buoyancy term and assumed to be linear with the temperature variations. As shown by Moreau (1990) and later used by Ben Hadid, Henry \& Kaddeche (1997a), Ben Hadid \& Henry (1997), Juel et al. (1999) and Kaddeche, Henry \& Ben Hadid (2003), in liquid metals the Lorentz body force induced by the magnetic field can be expressed as

$$
\vec{F}^{*}=\boldsymbol{J}^{*} \times \vec{B}_{0}=\sigma_{e}\left(\vec{E}^{*}+\boldsymbol{V}^{*} \times \vec{B}_{0}\right) \times \vec{B}_{0},
$$


where $J^{*}$ is the density of electric current, $\vec{E}^{*}=-\nabla^{*} \Phi^{*}$ is the electric field, $\Phi^{*}$ is the electric potential and $V^{*}$ is the flow velocity. The quantities with a star refer to dimensional quantities.

Using $H, H^{2} / v, v / H, \rho_{0} v^{2} / H^{2},(\nabla T)^{*} H, v B_{0}$ and $\sigma_{e} v B_{0} / H$ as scales for length, time, velocity, pressure, temperature, electric potential and electric current, respectively, the governing equations for the problem are

$$
\begin{gathered}
\frac{\partial \boldsymbol{V}}{\partial t}+(\boldsymbol{V} \cdot \nabla) \boldsymbol{V}=-\nabla P+\nabla^{2} \boldsymbol{V}+G r T \boldsymbol{e}_{y}+H a^{2} \boldsymbol{J} \times \boldsymbol{e}_{B_{0}} \\
\frac{\partial T}{\partial t}+(\boldsymbol{V} \cdot \nabla T)=\frac{1}{P r} \nabla^{2} T \\
\nabla \cdot \boldsymbol{V}=0 \\
\boldsymbol{J}=-\nabla \Phi+\boldsymbol{V} \times \boldsymbol{e}_{B_{0}}, \\
\nabla \cdot \boldsymbol{J}=0,
\end{gathered}
$$

where the dimensionless variables are the velocity vector $\boldsymbol{V}=(U, V, W)$, the pressure $P$, the temperature $T$, the electric potential $\Phi$ and the electric current density vector $\boldsymbol{J}$. $\boldsymbol{e}_{y}$ and $\boldsymbol{e}_{B_{0}}$ are unit vectors in the vertical direction and in the direction of $\vec{B}_{0}$, respectively. Combining the continuity equation for $\boldsymbol{J}(2.6)$ and Ohm's law (2.5), we obtain the dimensionless equation governing the electric potential:

$$
\nabla^{2} \Phi=\boldsymbol{e}_{B_{0}} \cdot(\nabla \times \boldsymbol{V}) .
$$

The boundary conditions applied on the planes $x= \pm l / 2$ and $y= \pm 1 / 2$ are:

- No-slip condition for the velocity, $\boldsymbol{V}=0$;

- Fixed temperature (thermally conductive walls);

- Zero current flux (electrically insulating walls), $\partial \Phi / \partial n=0$, where $n$ is the unit normal vector.

The governing system (2.2)-(2.7) depends on four non-dimensional parameters: the Hartmann number $H a=\left|B_{0}\right| H \sqrt{\sigma_{e} / \rho_{0} v}$, the Grashof number $G r=g \beta(\nabla T)^{*} H^{4} / v^{2}$, the Prandtl number $\operatorname{Pr}=v / \kappa$ and the transverse aspect ratio $l=L / H$.

\section{Basic flow}

As in Lyubimova et al. (2008), we look for a solution in which the velocity, the temperature deviation from the distribution which corresponds to the uniform longitudinal temperature gradient, and now additionally the electric potential do not depend on $z$ :

$$
\boldsymbol{V}=\boldsymbol{V}(x, y)=(\vec{U}(x, y), W(x, y)), \quad T=z+\Theta(x, y) \text { and } \Phi=\Phi(x, y) .
$$

Then the pressure is a linear function of $z$ (Lyubimova et al. 2008):

$$
P=G r y z+\Pi(x, y)+C z,
$$

where $C$ is a constant.

Substituting the expressions (3.1) and (3.2) into (2.2)-(2.7), we obtain:

$$
\begin{gathered}
\frac{\partial \vec{U}}{\partial t}+\left(\vec{U} \cdot \nabla_{s}\right) \vec{U}=-\nabla_{s} \Pi+\nabla_{s}^{2} \vec{U}+G r \Theta \boldsymbol{e}_{y}+H a^{2} \vec{f}, \\
\frac{\partial W}{\partial t}+\left(\vec{U} \cdot \nabla_{s}\right) W=-G r y-C+\nabla_{s}^{2} W+H a^{2} F_{z},
\end{gathered}
$$




$$
\begin{gathered}
\frac{\partial \Theta}{\partial t}+\left(\vec{U} \cdot \nabla_{s}\right) \Theta+W=\frac{1}{P r} \nabla_{s}^{2} \Theta, \\
\nabla_{s} \cdot \vec{U}=0, \\
\vec{F}=\left(-\nabla \Phi+\boldsymbol{V} \times \boldsymbol{e}_{B_{0}}\right) \times \boldsymbol{e}_{B_{0}}, \\
\nabla^{2} \Phi=\boldsymbol{e}_{B_{0}} \cdot(\nabla \times \boldsymbol{V}),
\end{gathered}
$$

where $\vec{F}=\left(\vec{f}, F_{z}\right), \vec{f}=\left(F_{x}, F_{y}\right), \vec{U}=(U, V), \nabla_{s}$ is the two-dimensional differentiation operator defined upon the plane $(x, y)$. To obtain the expression for $C$, we integrate (3.4) on the cross-section $S\left((x, y)\right.$ plane) and take mass conservation, $\int_{S} W \mathrm{~d} S=0$, into account. We obtain

$$
C=\frac{1}{S} \int_{S} \nabla_{s}^{2} W \mathrm{~d} S+\frac{H a^{2}}{S} \int_{S} F_{z} \mathrm{~d} S .
$$

Let us assume that $\boldsymbol{e}_{B_{0}}$ lies in the $(x, y)$ plane and define the angle $\alpha$ between the magnetic field direction and the $x$-axis. By introducing the stream function and the vorticity for the transversal flow which are such that

$$
U=\frac{\partial \psi}{\partial y}, \quad V=-\frac{\partial \psi}{\partial x}, \quad \Omega=(\nabla \times \boldsymbol{V})_{z},
$$

we can rewrite (3.3)-(3.9) in the form:

$$
\begin{gathered}
\frac{\partial \Omega}{\partial t}+\mathscr{J}(\Omega, \psi)=\nabla_{s}^{2} \Omega+G r \frac{\partial \theta}{\partial y}+H a^{2}\left(\frac{\partial^{2} \psi}{\partial x \partial y} \sin 2 \alpha+\frac{\partial^{2} \psi}{\partial y^{2}} \sin ^{2} \alpha+\frac{\partial^{2} \psi}{\partial x^{2}} \cos ^{2} \alpha\right), \\
\Omega=-\nabla_{s}^{2} \psi \\
\frac{\partial W}{\partial t}+\mathscr{J}(W, \psi)=-G r y-C+\nabla_{s}^{2} W+H a^{2}\left(\frac{\partial \Phi}{\partial y} \cos \alpha-\frac{\partial \Phi}{\partial x} \sin \alpha-W\right) \\
\frac{\partial \Theta}{\partial t}+\mathscr{J}(\Theta, \psi)+W=\frac{1}{P r} \nabla_{s}^{2} \Theta \\
\nabla_{s}^{2} \Phi=\left(\frac{\partial W}{\partial y} \cos \alpha-\frac{\partial W}{\partial x} \sin \alpha\right) \\
C=\frac{1}{S} \oint_{\Gamma} \frac{\partial W}{\partial n} \mathrm{~d} \Gamma+\frac{H a^{2}}{S} \int_{S}\left(\frac{\partial \Phi}{\partial y} \cos \alpha-\frac{\partial \Phi}{\partial x} \sin \alpha\right) \mathrm{d} S
\end{gathered}
$$

where $\mathscr{J}\left(q, q^{\prime}\right)=(\partial q / \partial x)\left(\partial q^{\prime} / \partial y\right)-(\partial q / \partial y)\left(\partial q^{\prime} / \partial x\right)$, and $\Gamma$ is the contour bounding the cross-section $S$. Finally, the boundary conditions become

$$
\left.\psi\right|_{\Gamma}=\left.\frac{\partial \psi}{\partial n}\right|_{\Gamma}=\left.\Theta\right|_{\Gamma}=\left.W\right|_{\Gamma}=\left.\frac{\partial \Phi}{\partial n}\right|_{\Gamma}=0 .
$$

The calculation of the basic flows, solutions of these equations, has been performed with the same numerical methods as in Lyubimova et al. (2008). The effect of the grid size has been tested and, for $l=1$, grids with $101 \times 101$ points for a vertical magnetic field at $\operatorname{Pr}=0,91 \times 91$ points for a horizontal magnetic field at $\operatorname{Pr}=0$, and $71 \times 71$ points for $\operatorname{Pr} \neq 0$ where the basic flow is weaker than for $\operatorname{Pr}=0$, were chosen as they correctly reproduced the asymptotic behaviours at large $\mathrm{Ha}$ (Ben Hadid \& Henry 1994, 1997). 


\subsection{Zero Prandtl number case}

According to Lyubimova et al. (2008), at $P r=0$ the problem (described in (3.11)(3.17)) admits a steady solution in which only the longitudinal component of the velocity is non-zero. The equations and boundary conditions are thus reduced to:

$$
\begin{gathered}
0=-G r y-C+H a^{2}\left(\frac{\partial \Phi}{\partial y} \cos \alpha-\frac{\partial \Phi}{\partial x} \sin \alpha-W\right)+\nabla_{s}^{2} W \\
\nabla_{s}^{2} \Phi=\left(\frac{\partial W}{\partial y} \cos \alpha-\frac{\partial W}{\partial x} \sin \alpha\right), \\
\left.W\right|_{\Gamma}=\left.\frac{\partial \Phi}{\partial n}\right|_{\Gamma}=0 .
\end{gathered}
$$

In this paper, we only consider horizontal $(\alpha=0)$ and vertical $(\alpha=\pi / 2)$ magnetic fields. In both cases, the solutions have well defined symmetries: the longitudinal velocity $W$ is odd with respect to $y$ and even with respect to $x$, and the electric potential $\Phi$ is even with respect to both $x$ and $y$ coordinates in the case $\alpha=0$ and odd with respect to both coordinates in the case $\alpha=\pi / 2$. The constant $C$ turns out to be equal to zero in both cases.

As in the absence of magnetic field (Lyubimova et al. 2008), the velocity of the flow described by (3.18)-(3.20) is proportional to $G r$. In the lower part of the cavity, the fluid moves in the direction of the applied temperature gradient, whereas in the upper part, it moves in the opposite direction.

The problem, described in (3.18)-(3.20), is solved numerically as indicated in Lyubimova et al. (2008). Figures 2 and 3 present the isolines of the velocity $W$ and the streamlines of the electric current $J$ in the cross-section for the cases of vertically aligned $(\alpha=\pi / 2$, figure 2$)$ and horizontally aligned ( $\alpha=0$, figure 3 ) magnetic fields. Note that the streamlines of $\boldsymbol{J}$ in the cross-section are plotted from the current stream function $\psi_{j}$ which is such that $\boldsymbol{J}=\nabla \times\left(-\psi_{j} \boldsymbol{e}_{z}\right)$.

In the case of the vertical magnetic field, the electric current corresponds to a large current loop occupying most part of the cavity and thin current loops near the top and bottom boundaries (figure $2 b, d$ ). At $H a=5$, the flow is similar to the one observed in the absence of magnetic field, and in the case of a strong magnetic field $(H a=100)$, the longitudinal flow is concentrated in jets near the corners of the cross-section whereas the core of the cavity is more quiescent (figure $2 a, c$ ).

In the case of the horizontal magnetic field, the electric current flows from the top and bottom towards the centre of the cavity along the vertical $y$-axis and then sidewards along the $x$-axis, before returning along the vertical walls, thus forming four symmetrical electric current loops. These currents have maxima in areas adjacent to the walls, and principally in the Hartmann layers perpendicular to the magnetic field direction (figure $3 b, d$ ). Concerning the longitudinal flow structure, when the Hartmann number gets large enough values, boundary layers with significant vertical gradients of velocity appear near the horizontal walls in the parallel layers. The velocity maxima are shifted towards the horizontal walls, and the flow becomes more and more independent of the transverse direction (the direction of the magnetic field) except close to the vertical walls in the thin Hartmann layers where strong gradients appear (figure $3 a, c$ ).

According to the simulations, a stronger magnetic field yields a weaker flow in both cases. Figure 4 presents the dependence of the maximal velocity on the Hartmann number for $l=1$ and for vertical and horizontal magnetic fields. For a strong enough 
(a)

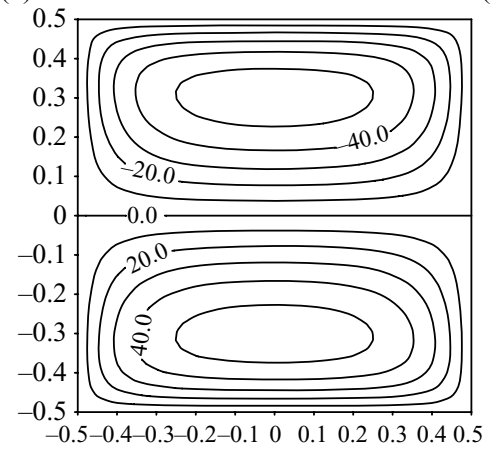

(b)

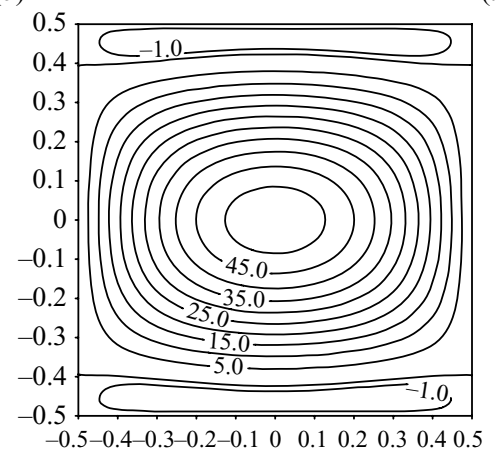

(c)

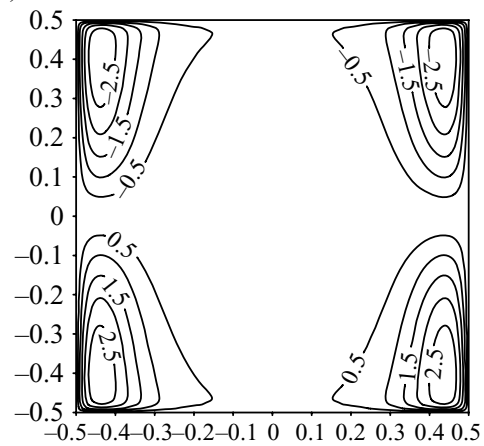

(d)

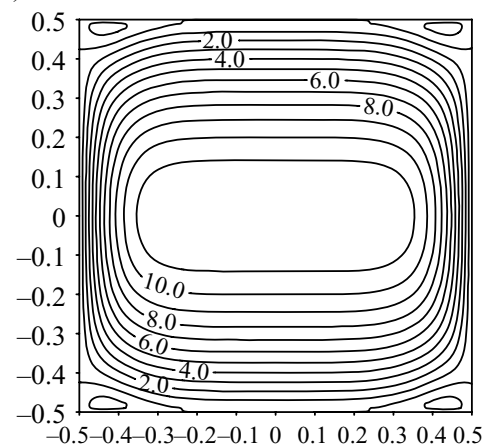

FIGURE 2. Isolines of the longitudinal velocity $(a, c)$ and streamlines of the electric current $(b, d)$ in the cross-section for the convective flow submitted to a vertical magnetic field at $\mathrm{Ha}=5(a, b)$ and $\mathrm{Ha}=100(c, d)$ and for $\mathrm{Pr}=0(\mathrm{Gr}=10000, l=1)$.

vertical magnetic field $(H a>10)$, the maximal velocity $W_{\max }$ obeys an hyperbolic law, $W_{\max } \sim H a^{-1}$, while the middle velocity $W_{\text {mid }}$ (i.e. measured on the $y$-axis) experiences a stronger decrease, $W_{\text {mid }} \sim \mathrm{Ha}^{-2}$. The braking effect is also well pronounced for the horizontal magnetic field, but it is less effective than for the vertical magnetic field.

All these results are in good agreement with those obtained for three-dimensional cavities by Ben Hadid \& Henry $(1994,1997)$.

\subsection{Non-zero Prandtl number case}

In the case of a non-zero Prandtl number, the problem does not admit plane-parallel flow solutions (Lyubimova et al. 2008) and the full system of governing equations, (3.11)-(3.17), has to be solved. The same numerical techniques as in Lyubimova et al. are used. The calculations are made for Prandtl numbers in the range $P r=0.01-0.15$ which corresponds to the liquid metals and for a cavity with a transverse aspect ratio $l=1$. As follows from the results of Lyubimova et al. for $H a=0$, this range of $\operatorname{Pr}$ is beyond the value $\operatorname{Pr}_{t}$ at which a sharp stabilization of the flow occurs, and the basic flow at $H a=0$ is characterized by the dominating role played by the four vortices located near the sidewalls which influence the structure of the longitudinal flow.

For both magnetic field orientations, a braking of the flow is observed with similar laws of velocity decrease as for $\operatorname{Pr}=0$. Figure 5 presents the isolines of the stream function and the temperature in the cross-section at $P r=0.1$ and $H a=100$ for the two magnetic field orientations $\alpha=\pi / 2$ and $\alpha=0$. The longitudinal flow and electric current structures in both cases are similar to those obtained for $\operatorname{Pr}=0$ (figure $2 c, d$ 
(a)

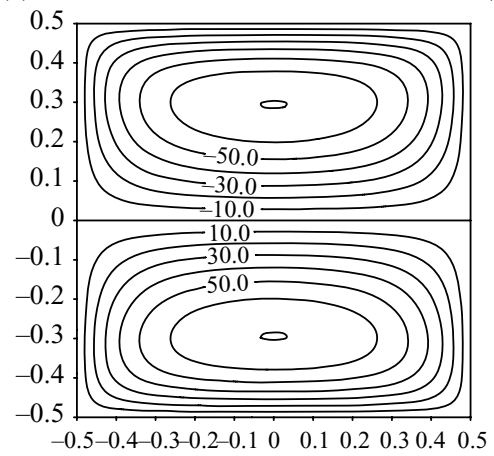

(b)

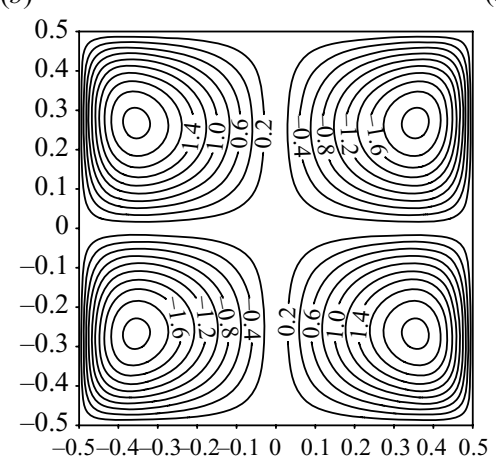

(c)

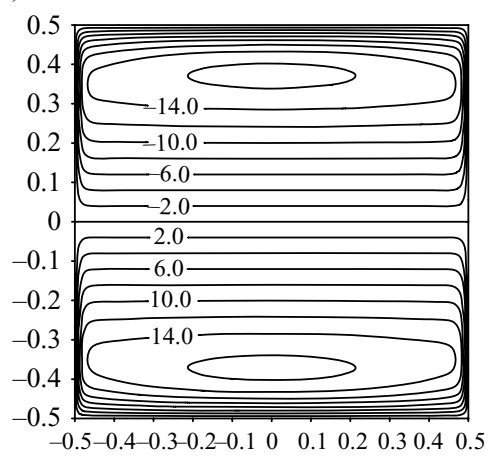

(d)

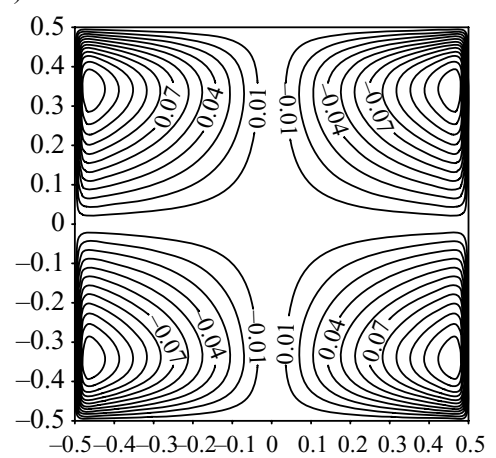

FIGURE 3. Isolines of the longitudinal velocity $(a, c)$ and streamlines of the electric current $(b, d)$ in the cross-section for the convective flow submitted to a horizontal magnetic field at $H a=5(a, b)$ and $\mathrm{Ha}=100(c, d)$ and for $\mathrm{Pr}=0(\mathrm{Gr}=10000, l=1)$.

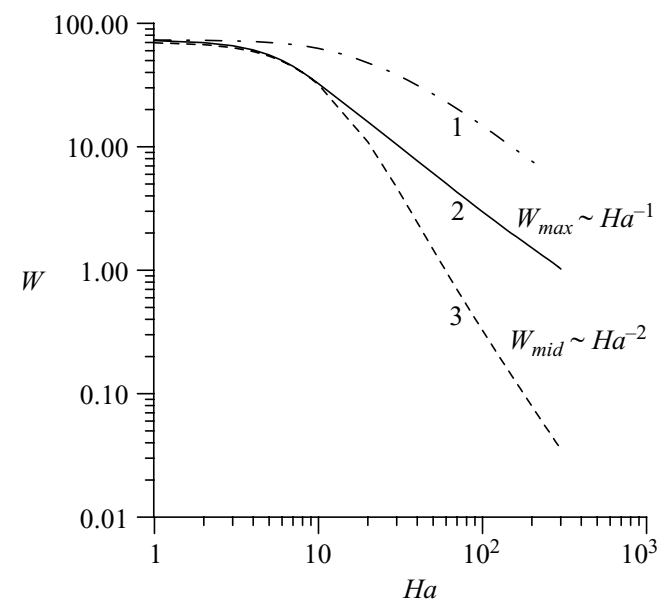

FIGURE 4. Variation with $H a$ of the longitudinal velocity maxima: (1) global maximum for a horizontal magnetic field, (2) global maximum and (3) maximum on the vertical mid-line for a vertical magnetic field $(G r=10000, \operatorname{Pr}=0, l=1)$.

and figure $3 c, d)$. This implies a smaller influence of the cross-section vortices on the longitudinal velocity in the presence of a magnetic field than without magnetic field. In fact the flow structure in the cross-section has evolved from the four-roll structure 
(a)

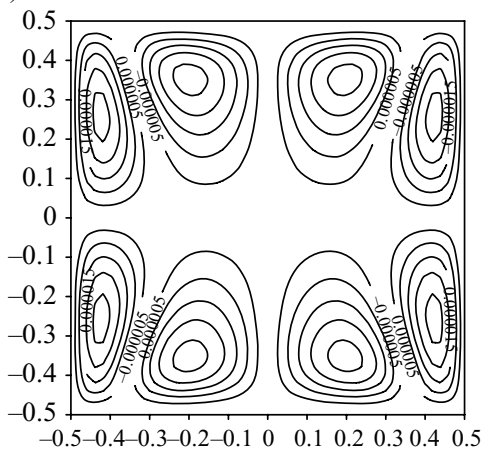

(b)

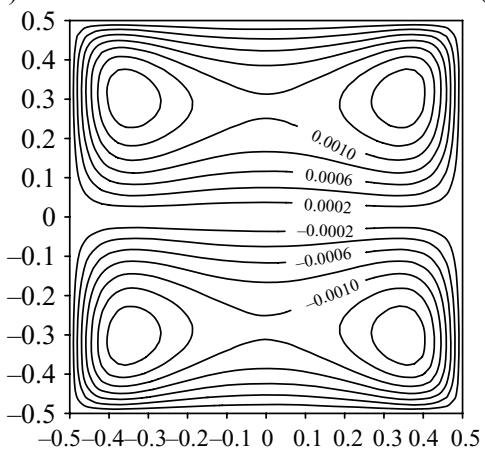

(c)

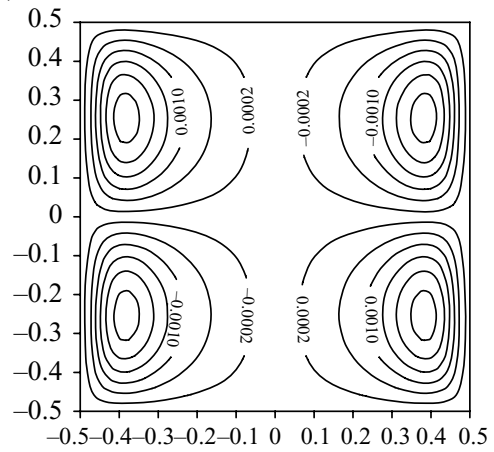

(d)

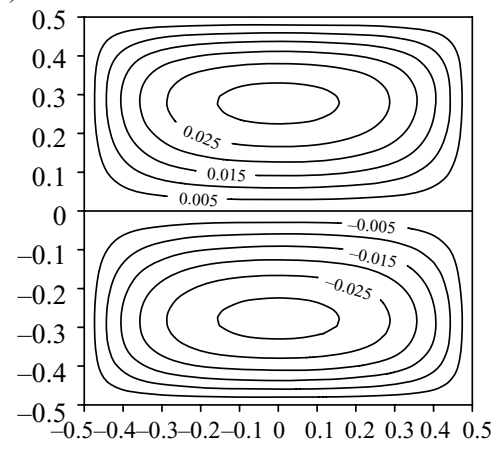

FIGURE 5. Streamlines of the velocity $(a, c)$ and isolines of the temperature $(b, d)$ in the cross-section for the convective flow submitted to a magnetic field, either vertical $(a, b)$ or horizontal $(c, d)$, at $H a=100$ and $\operatorname{Pr}=0.1(G r=10000, l=1)$.

obtained at $H a=0$. For both orientations of the magnetic field, the rolls are shifted towards the vertical walls, and in the case of the vertical magnetic field four additional rolls arise in the core (figure $5 a, c$ ). The temperature fields in the cross-section in the two cases are also different (figure $5 b, d$ ): they reflect the difference in longitudinal flow structures (figures $2 c$ and $3 c$ ).

\section{Stability analysis}

Let us study the stability of the solutions we have obtained. The magnetic field is still assumed to be in the $(x, y)$ plane. The velocity, electric potential, pressure, temperature and current density fields are now represented as the sum of the basic solution and infinitesimal perturbations:

$$
\boldsymbol{V}=\boldsymbol{V}_{0}+\boldsymbol{V}^{\prime}, \quad P=P_{0}+P^{\prime}, \quad T=T_{0}+\Theta^{\prime}, \quad \Phi=\Phi_{0}+\Phi^{\prime}, \quad \boldsymbol{J}=\boldsymbol{J}_{0}+\boldsymbol{J}^{\prime} .
$$

These perturbations can be chosen as normal modes in the $z$ direction:

$$
\left(\boldsymbol{V}^{\prime}, P^{\prime}, \Theta^{\prime}, \Phi^{\prime}, \boldsymbol{J}^{\prime}\right)=(\overrightarrow{\mathrm{v}}, p, \theta, \phi, \vec{j}) \mathrm{e}^{\mathrm{i} k z+\lambda t},
$$

where $k$ is the wave number in the $z$ direction and $\lambda=\lambda_{r}+\mathrm{i} \lambda_{i}$ is a complex growth rate.

After substitution of the expressions (4.1) and (4.2) into the system (2.2)-(2.7), linearization and projection onto the coordinate axes, we obtain the final governing 

equations and boundary conditions in the form:

$$
\begin{aligned}
& \lambda u=-\left(\vec{U}_{0} \nabla_{s}+\mathrm{i} k W_{0}\right) u-\left(\vec{u} \cdot \nabla_{s}\right) U_{0}-\frac{\partial p}{\partial x}+\left(\nabla_{s}^{2}-k^{2}\right) u \\
& +H a^{2}\left(\mathrm{i} k \phi \sin \alpha+\frac{1}{2} v \sin 2 \alpha-u \sin ^{2} \alpha\right), \\
& \lambda v=-\left(\vec{U}_{0} \cdot \nabla_{s}+\mathrm{i} k W_{0}\right) v-\left(\vec{u} \cdot \nabla_{s}\right) V_{0}-\frac{\partial p}{\partial y}+\left(\nabla_{s}^{2}-k^{2}\right) v+G r \theta \\
& +H a^{2}\left(-\mathrm{i} k \phi \cos \alpha+\frac{1}{2} u \sin 2 \alpha-v \cos ^{2} \alpha\right), \\
& \lambda w=-\left(\vec{U}_{0} \cdot \nabla_{s}+\mathrm{i} k W_{0}\right) w-\left(\vec{u} \cdot \nabla_{s}\right) W_{0}-\mathrm{i} k p+\left(\nabla_{s}^{2}-k^{2}\right) w \\
& +H a^{2}\left(\frac{\partial \phi}{\partial y} \cos \alpha-\frac{\partial \phi}{\partial x} \sin \alpha-w\right), \\
& \lambda \theta=-\left(\vec{U}_{0} \cdot \nabla_{s}+\mathrm{i} k W_{0}\right) \theta-\left(\vec{u} \cdot \nabla_{s}\right) \Theta_{0}-w+\frac{1}{P r}\left(\nabla_{s}^{2}-k^{2}\right) \theta, \\
& 0=\left(\nabla_{s}^{2}-k^{2}\right) \phi-\left(\mathrm{i} k u-\frac{\partial w}{\partial x}\right) \sin \alpha-\left(\frac{\partial w}{\partial y}-\mathrm{i} k v\right) \cos \alpha, \\
& 0=\frac{\partial u}{\partial x}+\frac{\partial v}{\partial y}+\mathrm{i} k w
\end{aligned}
$$

with at $x= \pm l / 2$ and $y= \pm 1 / 2$ :

$$
\left.\vec{u}\right|_{\Gamma}=\left.\frac{\partial \phi}{\partial n}\right|_{\Gamma}=\left.\theta\right|_{\Gamma}=0,
$$

where $\overrightarrow{\mathrm{v}}=(\vec{u}, w)$ with $\vec{u}=(u, v) . \boldsymbol{V}_{0}=\left(\vec{U}_{0}, W_{0}\right)$ with $\vec{U}_{0}=\left(U_{0}, V_{0}\right)$ and $T_{0}=z+\Theta_{0}$ are the velocity and temperature fields for the basic solution.

After discretization of the governing equations, a generalized eigenvalue problem is obtained:

$$
A \boldsymbol{X}=\lambda B \boldsymbol{X}
$$

which is solved as indicated in Lyubimova et al. (2008). Now the unknown vector $\boldsymbol{X}$ is such that $\boldsymbol{X}=(\overrightarrow{\mathrm{v}}, \theta, p, \phi)$, i.e. it contains the fields of the perturbations of velocity, temperature, pressure and electric potential. The stability computations were performed using grids with typically for $l=1,101 \times 101$ points for the cases at $P r=0$ with a vertical magnetic field, $91 \times 91$ points for the cases at $\operatorname{Pr}=0$ with a horizontal magnetic field and $71 \times 71$ points for the cases at $\operatorname{Pr} \neq 0$.

\subsection{Zero Prandtl number case}

According to Lyubimova et al. (2008), in the absence of magnetic field and at zero Prandtl number, a hydrodynamic steady instability mode related to the development of vortices at the boundary between the upper and lower counter flows is the most dangerous in a wide range of values of the aspect ratio $l$. The same steady mode remains the most dangerous for the case $P r=0$ when a magnetic field is applied. The results are given through curves showing the variation of the critical thresholds $G r_{c}$ and wave numbers $k_{c}$ with $H a$ for different aspect ratios $l$. These curves are presented in figures 6 and 7 for the vertical and horizontal magnetic fields, respectively.

The vertical magnetic field induces a strong stabilizing effect on the flow (figure $6 a$ ): even a small increase of $\mathrm{Ha}$ results in a significant increase of the critical Grashof 

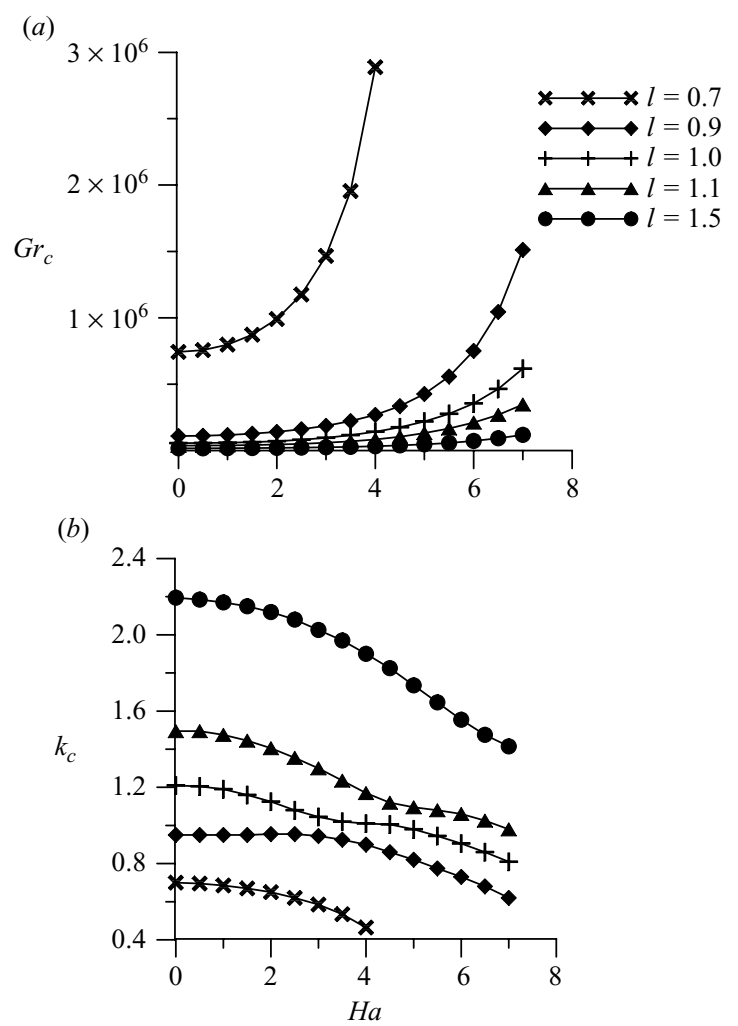

FIGURE 6. Variation of the critical Grashof number $G_{c}(a)$ and wave number $k_{c}(b)$ as a function of $\mathrm{Ha}$ for the convective flow submitted to a vertical magnetic field and for different channel widths $l(P r=0)$.

number. Moreover, the effect of stabilization is magnified by the decrease of the aspect ratio $l$. The wavelength of the most dangerous perturbations is increased with $\mathrm{Ha}$ (figure $6 b$ ). Such a strong increase of the thresholds (exponential laws) was previously obtained for the steady hydrodynamic modes in the case of an infinite layer $(l \rightarrow \infty)$ when a vertical magnetic field was applied (Kaddeche et al. 2003). A comparison with the results of Kaddeche et al. obtained for $\operatorname{Pr}=0.001$ shows that the increase of the thresholds for the confined layer is still larger than for the infinite layer. Exponential increases of the thresholds under vertical magnetic field were also obtained for three-dimensional cavities, either experimentally $(5 \times 1.3 \times 1$ cavity, Hof et al. 2005) or numerically $(4 \times 2 \times 1$ cavity, Henry et al. 2008b). In both cases, the increase of the thresholds was found to be smaller than for the infinite layer, and consequently also smaller than for the confined layer.

We may note that, in the confined layer, the hydrodynamic instability at $\operatorname{Pr}=0$ develops in the core of the section, i.e. in a zone where the flow evolves as $\mathrm{Ha}^{-2}$ similar to the evolution of the basic flow profile in the infinite layer. Moreover, it was shown in Lyubimova et al. (2008) that a similar development of the instability in the core of the section was observed for non-zero Prandtl numbers, until the strong increase of the thresholds at $P r_{t}$. From that, we can expect that in this $P r$ range, similar strong stabilizations by the vertical magnetic field can be obtained. 


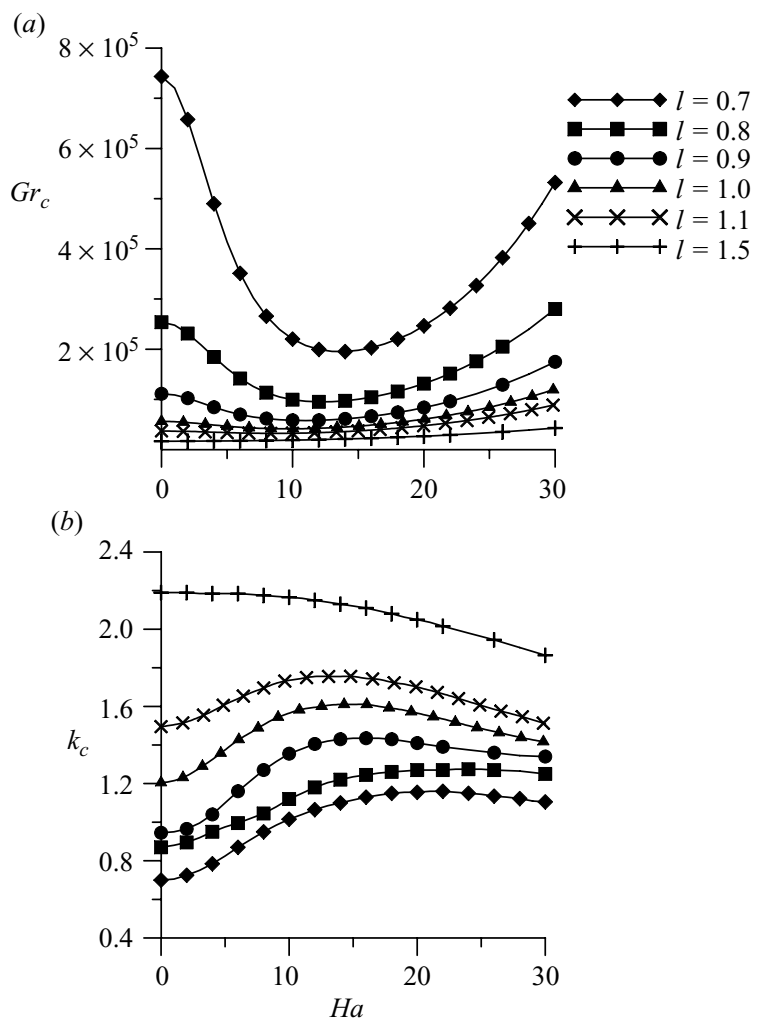

FIGURE 7. Variation of the critical Grashof number $G r_{c}(a)$ and wave number $k_{c}(b)$ as a function of $\mathrm{Ha}$ for the convective flow submitted to a horizontal magnetic field and for different channel widths $l(\operatorname{Pr}=0)$.

The horizontal magnetic field, on the contrary, can destabilize the flow in the range of small to moderate Hartmann number values: for small values of $l, G r_{c}$ decreases with the increase of $H a$, until it attains a minimum beyond which $G r_{c}$ begins to grow (figure $7 a$ ). This effect is magnified by the decrease of $l$. The Hartmann numbers corresponding to the minimal values of $G r_{c}$ decrease with the increase of the aspect ratio $l$. When the cavity is wide enough $(l \geqslant 1.4)$, there is no more minima in the curves and the horizontal magnetic field has a continuous stabilizing effect on the flow, but this effect is much weaker than that obtained with the vertical magnetic field. As seen from figure 7(b), for small values of $l$, the wave numbers of the neutral perturbations first increase with $\mathrm{Ha}$ until a maximum value and then start to decrease. When $l \geqslant 1.4$, the critical wave numbers decrease monotonously. We may recall that in the case of the infinite layer, the horizontal transverse magnetic field has no influence on the hydrodynamic thresholds (Kaddeche et al. 2003) whereas its influence on the thresholds in three-dimensional cavities (Hof et al. 2005; Henry et al. 2008b) is stabilizing but less efficient than the influence of the vertical magnetic field.

\subsection{Non-zero Prandtl number case}

The calculations in the non-zero Prandtl number case were performed in the range $\operatorname{Pr}=0.01-0.15$ and for a cavity with a transverse aspect ratio $l=1$. According to Lyubimova et al. (2008), all over this Prandtl number range, the hydrodynamic steady instability is still the most dangerous. But, for $l=1$, this Prandtl number range (which 

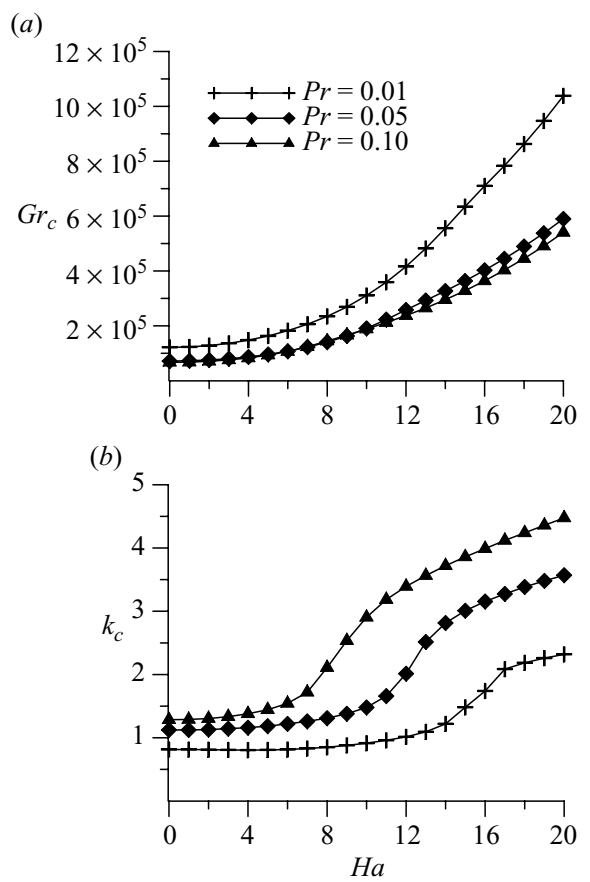

FIGURE 8. Variation of the critical Grashof number $G r_{c}(a)$ and wave number $k_{c}(b)$ as a function of $\mathrm{Ha}$ for the convective flow submitted to a vertical magnetic field and for different values of $\operatorname{Pr}(l=1)$.

is beyond $P r_{t}$ ) corresponds more precisely to an instability which develops near the side walls.

Both magnetic fields induce a stabilizing effect on the flow in the Prandtl number range covered by the computations (figures $8 a$ and $9 a$ ). This effect is, however, stronger for the vertical magnetic field than for the horizontal magnetic field. Moreover, for the chosen values of $P r$, the stabilizing effect obtained with the vertical field is smaller than that obtained at $\operatorname{Pr}=0$ for the same aspect ratio. In fact, the instability occurs here near the side walls, in a zone where, for the vertical magnetic field, the decrease of the flow, and thus the decrease of the shear responsible for this hydrodynamic instability, is smaller than in the core (progressive development of the longitudinal jets). We can also note that, differently from the case $P r=0$ where an increase of the critical threshold is associated to a decrease of the critical wave number, $k_{c}$ increases with $\mathrm{Ha}$ in the chosen range of $\operatorname{Pr}$ (figures $8 b$ and $9 b$ ).

$G r_{c}$ is also given as a function of $\mathrm{Pr}$ for $\mathrm{Ha}=0$ and $\mathrm{Ha}=5$ in figure 10. In the absence of magnetic field, the variation of $\mathrm{Gr}_{c}$ with $\mathrm{Pr}$ is non-monotonous in the Prandtl number range $P r=0.01-0.15$ (Lyubimova et al. 2008). As seen from figure 10, the vertical and horizontal magnetic fields, while increasing the thresholds, keep this non-monotonous variation of $G r_{c}$ with $P r$. Figure 10 also confirms that the vertical magnetic field is more efficient in stabilizing the flow than the horizontal magnetic field.

\section{Energy analyses}

Energy analyses are performed for $P r=0$ to characterize the variation of the thresholds when $\mathrm{Ha}$ is increased for both the vertical and horizontal magnetic fields. 

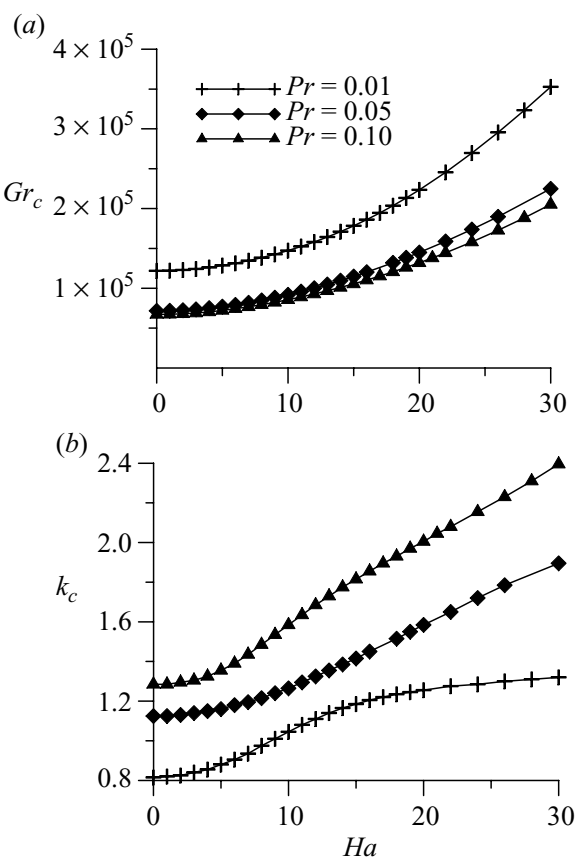

FiguRE 9. Variation of the critical Grashof number $G r_{c}(a)$ and wave number $k_{c}(b)$ as a function of $\mathrm{Ha}$ for the convective flow submitted to a horizontal magnetic field and for different values of $\operatorname{Pr}(l=1)$.

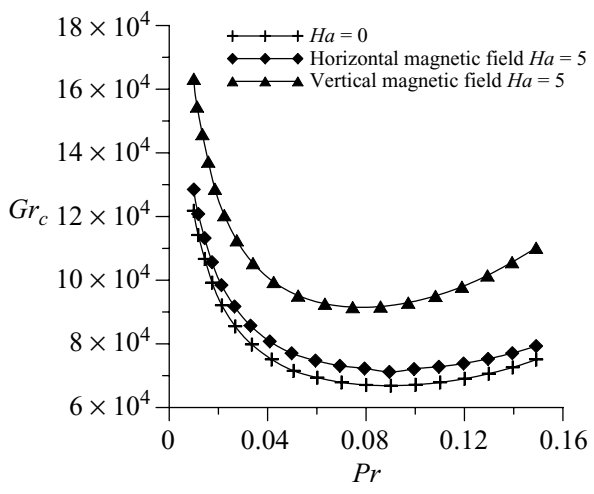

FIGURE 10. Variation of the critical Grashof number $G r_{c}$ as a function of $\operatorname{Pr}$ for the convective flow submitted to a magnetic field: crosses for $H a=0$, triangles for the vertical magnetic field at $H a=5$ and diamonds for the horizontal magnetic field at $H a=5(l=1)$.

The results are presented first as energy budgets and then as energetic contributions to the critical Grashof number.

\subsection{Fluctuating kinetic energy budgets}

As in Lyubimova et al. (2008), an equation giving the fluctuating kinetic energy budget can be derived from the linear stability equations, (4.3)-(4.5). In the case with magnetic field and for $\operatorname{Pr}=0$ (basic flow reduced to $W_{0}$, no temperature perturbations), the 


\begin{tabular}{|c|c|c|c|c|c|c|c|}
\hline \multicolumn{4}{|c|}{$l=0.7$} & \multicolumn{4}{|c|}{$l=1$} \\
\hline $\mathrm{Ha}$ & $E_{s x}^{\prime}$ & $E_{s y}^{\prime}$ & $E_{m}^{\prime}$ & $\mathrm{Ha}$ & $E_{s x}^{\prime}$ & $E_{s y}^{\prime}$ & $E_{m}^{\prime}$ \\
\hline 0 & -0.0584 & 1.0584 & 0 & 0 & -0.0306 & 1.0306 & 0 \\
\hline 1 & -0.0335 & 1.0612 & -0.0277 & 1 & -0.0277 & 1.0352 & -0.0075 \\
\hline 2 & -0.0349 & 1.0697 & -0.0348 & 2 & -0.0282 & 1.0474 & -0.0192 \\
\hline 3 & -0.0372 & 1.0841 & -0.0469 & 3 & -0.0300 & 1.0661 & -0.0361 \\
\hline 4 & -0.0406 & 1.1066 & -0.0660 & 5 & -0.0334 & 1.1066 & -0.0732 \\
\hline & & & & 6 & -0.0361 & 1.1269 & -0.0908 \\
\hline & & & & 7 & -0.0394 & 1.1460 & -0.1066 \\
\hline
\end{tabular}

TABLE 1. Fluctuating kinetic energy contributions: $E_{s x}^{\prime}$ and $E_{s y}^{\prime}$, production by shear; $E_{m}^{\prime}$, dissipation by magnetic forces $(l=0.7$ and $l=1$, vertical magnetic field, $\operatorname{Pr}=0)$.

\begin{tabular}{|c|c|c|c|c|c|c|c|}
\hline \multicolumn{4}{|c|}{$l=0.7$} & \multicolumn{4}{|c|}{$l=1.5$} \\
\hline$H a$ & $E_{s x}^{\prime}$ & $E_{s y}^{\prime}$ & $E_{m}^{\prime}$ & $\mathrm{Ha}$ & $E_{s x}^{\prime}$ & $E_{s y}^{\prime}$ & $E_{m}^{\prime}$ \\
\hline 0 & -0.0487 & 1.0487 & 0 & 0 & -0.0199 & 1.0199 & 0 \\
\hline 5 & -0.0348 & 1.0707 & -0.0359 & 5 & -0.0258 & 1.0971 & -0.0713 \\
\hline $14(\min )$ & -0.0376 & 1.2259 & -0.1883 & 12 & -0.0374 & 1.2612 & -0.2238 \\
\hline 24 & -0.0306 & 1.2973 & -0.2667 & 24 & -0.0237 & 1.2881 & -0.2644 \\
\hline
\end{tabular}

TABLE 2. Fluctuating kinetic energy contributions: $E_{s x}^{\prime}$ and $E_{s y}^{\prime}$, production by shear; $E_{m}^{\prime}$, dissipation by magnetic forces $(l=0.7$ and $l=1.5$, horizontal magnetic field, $\operatorname{Pr}=0)$.

normalized kinetic energy budgets at thresholds can be written as

$$
E_{s x}^{\prime}+E_{s y}^{\prime}+E_{m}^{\prime}=1
$$

where $E_{s x}^{\prime}=-\mathscr{R} e\left(\int_{S} u \frac{\partial W_{0}}{\partial x} w^{*} \mathrm{~d} x \mathrm{~d} y\right) /\left|E_{d}\right|$ and $E_{s y}^{\prime}=-\mathscr{R} e\left(\int_{S} v \frac{\partial W_{0}}{\partial y} w^{*} \mathrm{~d} x \mathrm{~d} y\right) /\left|E_{d}\right|$ are the productions of fluctuating kinetic energy by shear of the basic flow $\left(E_{s x}^{\prime}+\right.$ $\left.E_{s y}^{\prime}=E_{s}^{\prime}\right)$, and $E_{m}^{\prime}=\mathscr{R} e\left(H a^{2} \int_{S}\left[\left(-\nabla \phi+\overrightarrow{\mathrm{v}} \times \boldsymbol{e}_{B_{0}}\right) \times \boldsymbol{e}_{B_{0}}\right] \overrightarrow{\mathrm{v}}^{*} \mathrm{~d} x \mathrm{~d} y\right) /\left|E_{d}\right|$ is the dissipation of fluctuating kinetic energy by magnetic forces, all these terms being normalized by the viscous dissipation of fluctuating kinetic energy $E_{d}$.

In tables $1-2$, the different terms of the energy budget are given as a function of $H a$ for situations at their critical thresholds, in the case of a vertical magnetic field $(l=0.7$ and $l=1)$ and in the case of a horizontal magnetic field $(l=0.7$ and $l=1.5)$. In any case, the dominant destabilizing term is $E_{s y}^{\prime}$, the production of fluctuating kinetic energy by the vertical shear of the basic flow. $E_{s x}^{\prime}$ which is connected to the horizontal shear, is slightly stabilizing. It is also really negligible with regard to $E_{s y}^{\prime}$ ( $4 \%$ at the most for the cases presented in tables 1-2). Concerning the stabilizing magnetic term $E_{m}^{\prime}$, its intensity increases with the increase of $H a$ but begins to level off for $H a$ around 20. In any case, the values of $\left|E_{m}^{\prime}\right|$ remain moderate, reaching $25 \%$ of $E_{s y}^{\prime}$ at the most for the horizontal magnetic field where values of $\mathrm{Ha}$ up to 24 have been used. As $E_{s x}^{\prime}$ remains small, the increase of $E_{s y}^{\prime}$ with $H a$ from 1 is similar to the increase of $\left|E_{m}^{\prime}\right|$ from 0.

\subsection{Energetic contributions to the critical Grashof number}

The previous analysis has shown the relative importance of the terms in the fluctuating kinetic energy budget and how they evolve when a magnetic field is applied. But this analysis has not clarified the very different behaviours obtained with the two directions 


\begin{tabular}{cccc}
\multicolumn{3}{c}{$l=0.7$} \\
\hline$H a$ & $R_{1}$ & $R_{2}$ & $G r_{c} / G r_{c_{0}}$ \\
1 & 1.0438 & 1.0277 & 1.0727 \\
2 & 1.2874 & 1.0348 & 1.3322 \\
3 & 1.8833 & 1.0469 & 1.9716 \\
4 & 3.6430 & 1.0660 & 3.8835
\end{tabular}

\begin{tabular}{cccc}
\multicolumn{4}{c}{$l=1$} \\
\hline$H a$ & $R_{1}$ & $R_{2}$ & $G r_{c} / G r_{c_{0}}$ \\
1 & 1.0522 & 1.0075 & 1.0601 \\
2 & 1.2426 & 1.0192 & 1.2665 \\
3 & 1.6498 & 1.0361 & 1.7093 \\
5 & 2.1316 & 1.0732 & 2.2877 \\
6 & 5.8161 & 1.0908 & 6.3442 \\
7 & 9.9654 & 1.1066 & 11.028
\end{tabular}

TABLE 3. Characterization of the stabilization by a vertical magnetic field for $l=0.7$ and $l=1$ through the decomposition of the normalized critical Grashof number into two factors $R_{1}$ and $R_{2}$, respectively, related to the shear energy and the magnetic energy $(\operatorname{Pr}=0)$.

\begin{tabular}{|c|c|c|c|c|c|c|c|}
\hline \multicolumn{4}{|c|}{$l=0.7$} & \multicolumn{4}{|c|}{$l=1.5$} \\
\hline $\mathrm{Ha}$ & $R_{1}$ & $R_{2}$ & $G r_{c} / G r_{c_{0}}$ & $H a$ & $R_{1}$ & $R_{2}$ & $G r_{c} / G r_{c_{0}}$ \\
\hline 5 & 0.53712 & 1.0359 & 0.55638 & 5 & 0.96035 & 1.0713 & 1.0288 \\
\hline 14 (min.) & 0.22103 & 1.1883 & 0.26264 & 12 & 0.96575 & 1.2238 & 1.1819 \\
\hline 24 & 0.34711 & 1.2667 & 0.43967 & 24 & 1.5076 & 1.2644 & 1.9062 \\
\hline
\end{tabular}

TABLE 4. Characterization of the stabilization by a horizontal magnetic field for $l=0.7$ and $l=1.5$ through the decomposition of the normalized critical Grashof number into two factors $R_{1}$ and $R_{2}$, respectively, related to the shear energy and the magnetic energy $(\operatorname{Pr}=0)$.

of the magnetic field for $P r=0$. Another approach is to express the critical Grashof number in terms of energetic contributions. For that, noting that, for $\operatorname{Pr}=0, E_{s}^{\prime}$ (through $W_{0}$ ) is proportional to $\operatorname{Gr}\left(E_{s}^{\prime}=G r E_{s}^{\prime \prime}\right.$ corresponding to $\left.W_{0}=G r w_{0}\right)$, we can use (5.1) applied for $H a$ non zero and $H a=0$ (subscript 0 ) to derive an expression for $G r_{c} / G r_{c_{0}}$ :

$$
\frac{G r_{c}}{G r_{c_{0}}}=R_{1} R_{2}
$$

where

$$
R_{1}=\left(\frac{E_{s_{0}}^{\prime \prime}}{E_{s}^{\prime \prime}}\right), R_{2}=1-E_{m}^{\prime}
$$

As $E_{m}^{\prime}<0$, the action of the magnetic field will increase $G r_{c}$ by the increase of $R_{2}$ in connection with the Lorentz forces. The action of the magnetic field will also modify the velocity profile and the perturbations leading to a change of $E_{s}^{\prime \prime}$ and thus to a change of $R_{1}$ and $G r_{c}$. Note that $R_{1}$ and $R_{2}$ are equal to 1 at $H a=0$.

The evolution with $\mathrm{Ha}$ of $R_{1}, R_{2}$ and $G r_{c} / G r_{c_{0}}=R_{1} R_{2}$ are given in tables 3-4 for the same cases as in the previous section. In any case, $R_{2}$ increases with $H a$, but in a moderate way (values only slightly departing from 1), so that the evolution of the thresholds with $H a$ is principally connected to the evolution of $R_{1}$. For the vertical magnetic field, $R_{2}$ really remains close to 1 and the strong increase of the threshold with $H a$ is explained by the strong increase of $R_{1}$ (table 3 ). This type of behaviour has already been obtained for an infinite layer $(l \rightarrow \infty)$ under vertical magnetic field by Kaddeche et al. (2003). For the horizontal magnetic field in a cavity with $l=0.7$, the initial decrease and further increase of the threshold with $H a$ is also explained by a similar evolution found for $R_{1}$ (table 4). The evolution of $R_{2}$, although not dominant, 


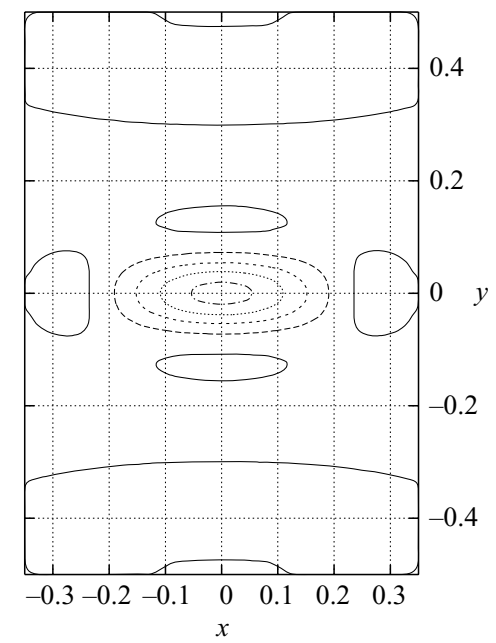

FIGURE 11. Isolines of the shear energy $S_{y G}=\mathscr{R} e\left(-\frac{\partial w_{0}}{\partial y} v w^{*}\right) /\left|E_{d}\right|$ at threshold for $l=0.7$ and $\mathrm{Ha}=0(\mathrm{Pr}=0)$. Solid lines indicate the zero isoline, and the increase between successive isolines is $10^{-5}$.

has a greater influence for the horizontal magnetic field. For instance, for a cavity with $l=1.5$ (table 4 ), the small initial decrease of $R_{1}$ is hidden by the increase of $R_{2}$, so that a continuous increase of the threshold with $\mathrm{Ha}$ is obtained.

All these indicate that it is the evolution of the energy generated by shear which plays an important role in the evolution of the thresholds. Moreover as we have shown that $E_{s x}^{\prime} \ll E_{s y}^{\prime}$, the main influence comes from $E_{s y}^{\prime \prime}=\left(\int_{S} \mathscr{R} e\left(-\frac{\partial w_{0}}{\partial y} v w^{*}\right) \mathrm{d} x \mathrm{~d} y\right) /\left|E_{d}\right|$. We will now analyse this term by decomposing it into its main components, and thus define three quantities, $S_{y G}=\mathscr{R} e\left(-\frac{\partial w_{0}}{\partial y} v w^{*}\right) /\left|E_{d}\right|, M_{G}=\left(-\frac{\partial w_{0}}{\partial y}\right)$ and $F=\mathscr{R} e\left(v w^{*}\right) /\left|E_{d}\right|$, which are such that

$$
E_{s y}^{\prime \prime}=\left(\int_{S} S_{y G} \mathrm{~d} x \mathrm{~d} y\right)=\left(\int_{S} M_{G} F \mathrm{~d} x \mathrm{~d} y\right) .
$$

$S_{y G}$ is the energy field due to the shear $\frac{\partial w_{0}}{\partial y}$ which gives $E_{s y}^{\prime \prime}$ by integration on the section, $M_{G}$ is the factor depending on the basic flow (shear at constant $G r$ ) and $F$ is the factor depending on the fluctuating flow (note that compared to Lyubimova et al. 2008, we have $S_{y}=G r S_{y G}$ and $M=G r M_{G}$ ). We will also introduce two other quantities related to the norm of the fluctuating velocity components, $F_{w}=\sqrt{\left(w w^{*}\right) /\left|E_{d}\right|}$ and $F_{v}=\sqrt{\left(v v^{*}\right) /\left|E_{d}\right|}$. This analysis of $E_{s y}^{\prime \prime}$ will be done for $l=0.7$, as the influence of the magnetic field direction is clearly important in this case.

The isovalues of $S_{y G}$ for $l=0.7$ and $H a=0$ are first shown in figure 11 . We see that the energy is concentrated in a zone around the centre point of the section, and that the values outside this zone are really small and then clearly negligible. Because of this concentration of the energy, and as it would be difficult to clearly analyse the evolution of the different terms with $\mathrm{Ha}$ from two-dimensional plots, we have chosen to characterize these terms from one-dimensional profiles taken along the vertical direction $y$ in the centre of the section. The vertical profiles of $S_{y G}$ for $l=0.7$ are given in figure 12 for different values of $\mathrm{Ha}$, in the case of the vertical magnetic field (figure 12a) and in the case of the horizontal magnetic field (figure 12b). These plots show the systematic decrease of $S_{y G}$ for the vertical magnetic field (figure 12a) and 

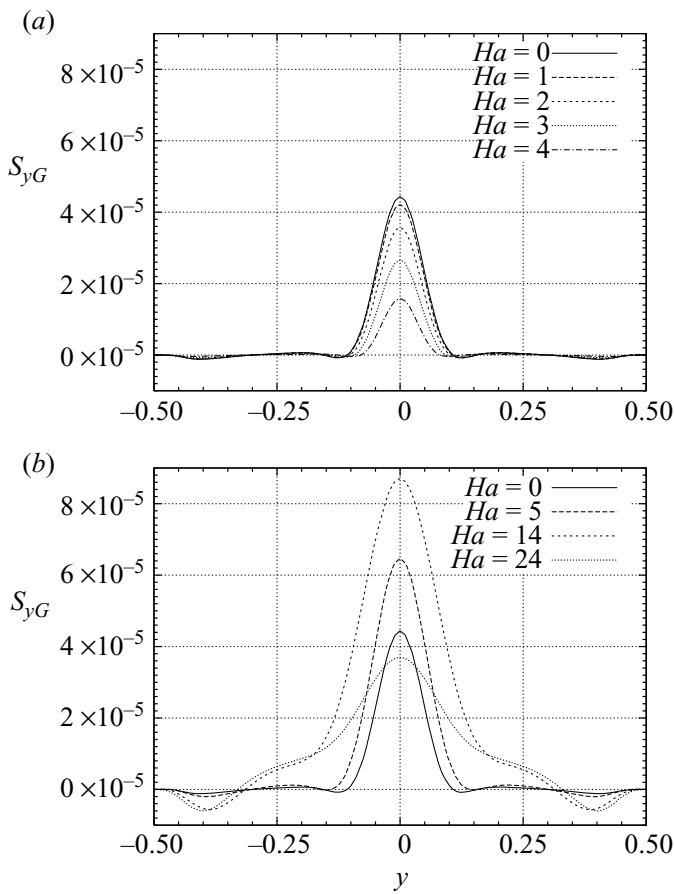

FigURE 12. Vertical profiles of the shear energy $S_{y G}=\mathscr{R} e\left(-\frac{\partial w_{0}}{\partial y} v w^{*}\right) /\left|E_{d}\right|$ at threshold in the centre of the cavity for a vertical magnetic field $(a)$ and a horizontal magnetic field $(b)(l=0.7$, $\operatorname{Pr}=0)$.

the initial increase and then decrease of $S_{y G}$ for the horizontal magnetic field (figure $12 b$ ). These variations are clearly connected to what was observed for $R_{1}$ in tables 3-4, having in mind the definition of $R_{1}$ (defined in (5.3)). In the same way, we now plot the vertical profiles of $M_{G}$ and $F$ in figure 13, and those of $F_{w}$ and $F_{v}$ in figure 14. In figure $13(a, b)$, we see that the profiles of $M_{G}$ (shear at constant $G r$ ) decrease as $\mathrm{Ha}$ is increased for the two directions of the magnetic field, this effect being quicker for the vertical field, but more pronounced for the horizontal field because of the higher values of $\mathrm{Ha}$. In fact, it is the evolution of $F$ given in figure $13(c, d)$ which is mainly responsible for what was observed on $S_{y G}$. The last step is to see the role of the fluctuating velocity components in the evolution of $F$. Figure $14(a, b)$ shows that $F_{w}$ principally decreases as $H a$ is increased for the two directions of the magnetic field (except at small $\mathrm{Ha}$ where a small increase is obtained for the horizontal field) whereas, as shown in figure $14(c, d), F_{v}$ has different behaviours for the two directions of the field: it decreases for the vertical magnetic field, but increases for the horizontal field.

We have thus seen that for the vertical magnetic field all the quantities $F_{w}, F_{v}, F$ and $M_{G}$ decrease as $H a$ is increased, leading to the decrease of $S_{y G}$ and $E_{s y}^{\prime \prime}$, and then to the increase of $R_{1}$. Among these quantities, the product of the velocity fluctuations normalized by the dissipation, represented by $F$, and more intimately the normalized vertical velocity fluctuation seem to have the more important influence. Concerning the horizontal magnetic field, the normalized vertical velocity fluctuation also plays a crucial role. Its strong initial increase as $H a$ is increased (observed through $F_{v}$ ) induces an initial increase of $F$ and $S_{y G}$, despite the decrease of $F_{w}$ and $M_{G}$, and 
(a)

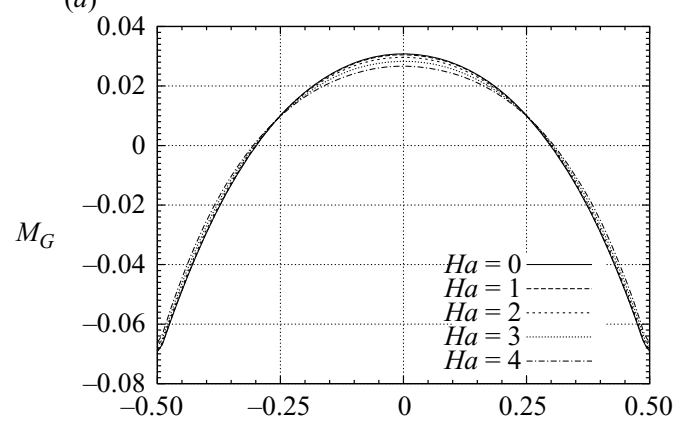

(b)

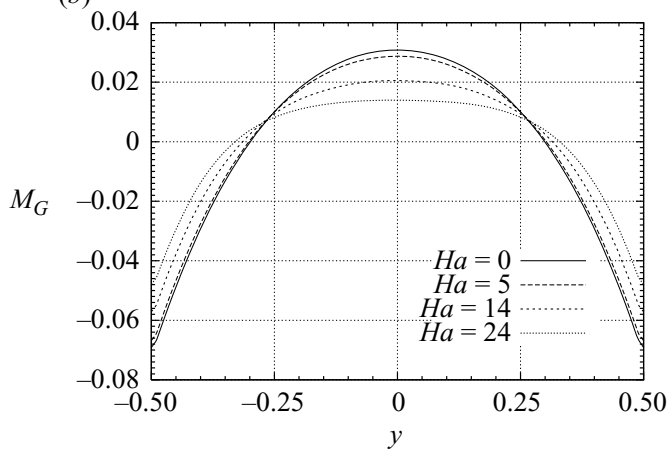

(c)

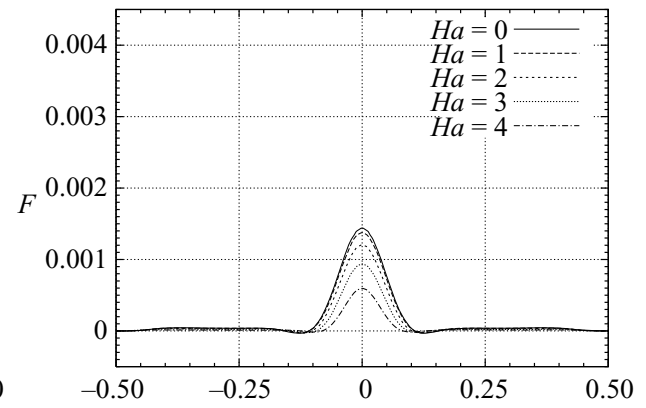

(d)

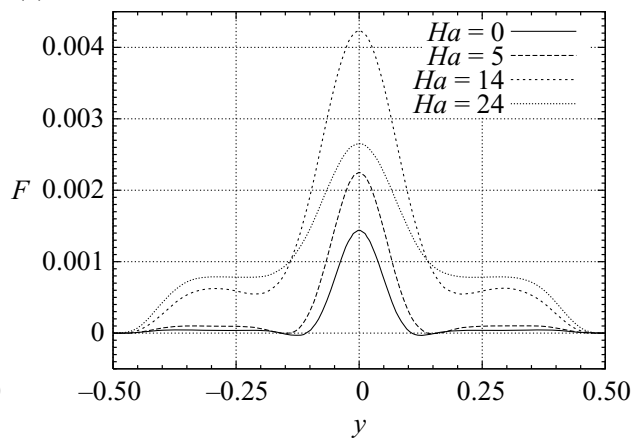

FIGURE 13. Vertical profiles of $M_{G}=\left(-\frac{\partial w_{0}}{\partial y}\right)(a, b)$ and $F=\mathscr{R} e\left(v w^{*}\right) /\left|E_{d}\right|(c, d)$ at threshold in the centre of the cavity for a vertical magnetic field $(a, c)$ and a horizontal magnetic field $(b, d)$ $(l=0.7, \operatorname{Pr}=0)$.

is then responsible for the initial decrease of $R_{1}$. For larger $H a$, the increase of $F_{v}$ weakens and the general decrease of the other quantities induces an increase of $R_{1}$.

In conclusion, we have shown that the evolution of the thresholds with the increase of $\mathrm{Ha}$ is principally connected to the evolution of the fluctuating energy generated by shear along the vertical direction. The evolution of this energy is, as expected, influenced by the modification of the velocity profiles by the magnetic field, but it has been shown that the main factor is in fact the modification of the fluctuating velocity field, and before all the modification of the fluctuating vertical velocity.

\section{Conclusion}

The effect of a magnetic field on the steady convective flow driven by a longitudinal temperature gradient in a horizontal channel of rectangular cross-section and on the stability of this flow has been numerically studied.

The convective flow calculations have shown that the general type of steady circulation obtained in the presence of a magnetic field is the same as in the absence of magnetic field: in the lower part of the channel the fluid moves in the direction of the imposed temperature gradient and in the upper part it moves in the opposite direction. The intensity of the flow and the velocity distribution over the cross-section, however, strongly depend on the magnetic field strength and direction.

In the case of a sufficiently strong vertical magnetic field, the convective flow is concentrated near the cross-section corners such that actually, instead of two counter flows there are four horizontal jets, two of them (the lower ones) coinciding with the 

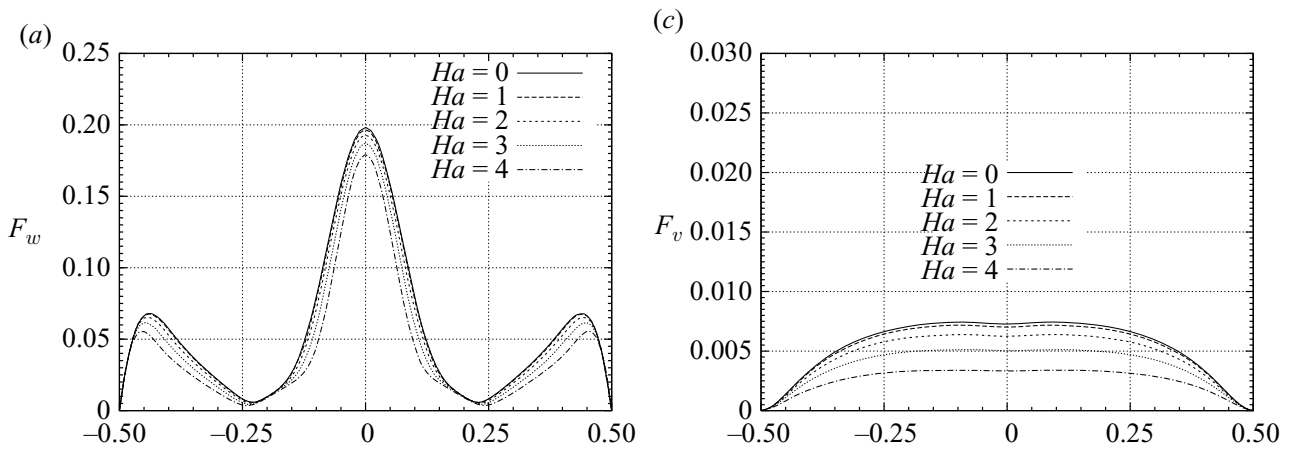

(b)

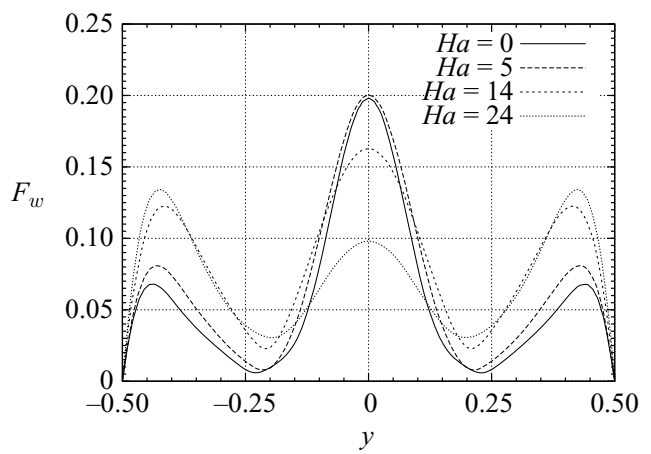

(d)

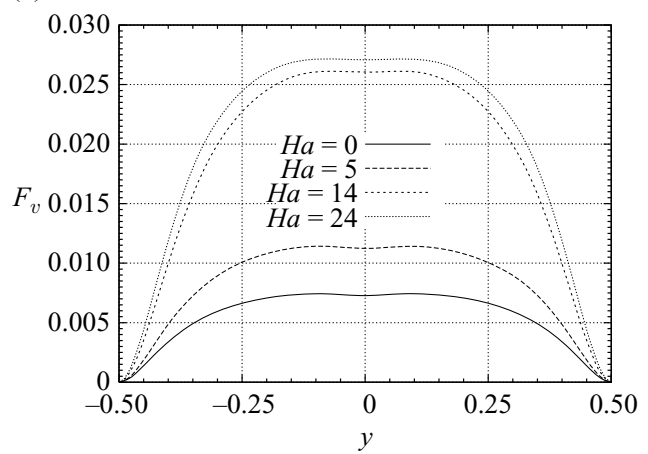

FIGURE 14. Vertical profiles of $F_{w}=\sqrt{\left(w w^{*}\right) /\left|E_{d}\right|}(a, b)$ and $F_{v}=\sqrt{\left(v v^{*}\right) /\left|E_{d}\right|}(c, d)$ at threshold in the centre of the cavity for a vertical magnetic field $(a, c)$ and a horizontal magnetic field $(b, d)(l=0.7, \operatorname{Pr}=0)$.

direction of the applied temperature gradient and the two others (the upper ones) moving in the opposite direction. The flow intensity is subject to a fast decrease with the growth of the magnetic field strength. Characteristic laws of variation can be obtained which, due to the concentration of the flow in jets near the corners, turn out to be different for the core flow and the jet flow, and proportional, respectively, to $H a^{-2}$ and $H a^{-1}$.

The effect of the horizontal magnetic field on the steady convective flow is different. A braking of the flow is obtained, but less effective than for the vertical magnetic field. The changes of the flow structure are also not so sharp: we can principally mention the decrease of the horizontal gradients of velocity in the central zone and the growth of the gradients in the boundary layers which develop along the walls.

The linear stability analysis of these convective flows submitted to a magnetic field has been performed for a Prandtl number $\mathrm{Pr}$ equal to 0 and different cross-section widths, and for $\mathrm{Pr}$ in the range $0.01-0.15$ in the case of a square cross-section. Steady perturbations with a finite wavelength appear to be the most dangerous in this parameter range, and, as expected from the decrease of the flow intensity and the energy loss by Joule dissipation, the magnetic field has a stabilizing effect on these perturbations in most part of the parameter range, the vertical magnetic field, with exponential laws of stabilization, being more effective than the horizontal field. An unexpected destabilizing influence of the magnetic field has however been obtained for zero Prandtl number in the case of the horizontal magnetic field. The decrease of the instability thresholds which is induced occurs as soon as the magnetic field is 
applied until moderate values of $H a$ and the effect is particularly strong when the width of the channel is less than its height. This destabilizing effect is still present, but weak, for a channel of square cross-section, and it vanishes quickly when the width of the channel is further increased.

The analysis at threshold of the kinetic energy budget associated with the most dangerous perturbations has shown that the dominant destabilizing contribution comes in any case from the vertical shear of the longitudinal velocity, and that the variations of the thresholds observed when a magnetic field is applied are principally connected to the evolution of this shear term, and not much to the stabilizing magnetic energy term. More precisely, in this shear term, the variation at threshold of the product of the scaled velocity disturbances is a key factor: a strong decrease of this product is found when $\mathrm{Ha}$ is increased in the case of the vertical magnetic field at $P r=0$ which, combined with the natural decrease of the basic flow shear with increasing $\mathrm{Ha}$ at constant $\mathrm{Gr}$, explains the strong exponential increase of the thresholds found in this case, whereas an increase of this product with $\mathrm{Ha}$ in the case of the horizontal magnetic field at $\mathrm{Pr}=0$ explains the unexpected initial decrease of the thresholds.

This work was funded by a fellowship from the Région Rhônes-Alpes (A.P.).

\section{REFERENCES}

Alboussière, T., Garandet, J. P. \& Moreau, R. 1993 Buoyancy-driven convection with a uniform magnetic field. Part 1. Asymptotic analysis. J. Fluid Mech. 253, 545-563.

Alboussière, T., Garandet, J. P. \& Moreau, R. 1996 Asymptotic analysis and symmetry in MHD convection. Phys. Fluids 8 (8), 2215-2226.

Baumgartl, J., Hubert, A. \& Müller, G. 1993 The use of magnetohydrodynamic effects to investigate fluid flow in electrically conducting melts. Phys. Fluids A 5 (12), 3280-3289.

Baumgartl, J. \& Müller, G. 1992 Calculation of the effects of magnetic field damping on fluid flow - comparison of magnetohydrodynamic models of different complexity. In Proceedings of the Eighth European Symposium on Materials and Fluid Sciences in Microgravity (ESA SP333), pp. 161-164. ESA Publication Division c/o ESTEC, Noordwijk, The Netherlands.

Ben Hadid, H. \& Henry, D. 1994 Numerical simulation of convective three-dimensional flows in a horizontal Bridgman configuration under the action of a constant magnetic field. In Proceedings of the Second Intl Conf. on Energy Transfer in MagnetoHydroDynamic Flows, Aussois, France, vol. 1, pp. 47-56. MHD Pamir Publications, Grenoble.

Ben Hadid, H. \& Henry, D. 1996 Numerical simulations of convective three-dimensional flows in a horizontal cylinder under the action of a constant magnetic field. J. Cryst. Growth 166, 436-445.

Ben Hadid, H. \& Henry, D. 1997 Numerical study of convection in the horizontal Bridgman configuration under the action of a constant magnetic field. Part 2. Three-dimensional flow. J. Fluid Mech. 333, 57-83.

Ben Hadid, H., Henry, D. \& Kaddeche, S. 1997a Numerical study of convection in the horizontal Bridgman configuration under the action of a constant magnetic field. Part 1. Two-dimensional flow. J. Fluid Mech. 333, 23-56.

Ben Hadid, H., Henry, D. \& Touihri, R. $1997 b$ Unsteady three-dimensional buoyancy-driven convection in a circular cylindrical cavity and its damping by magnetic field. J. Cryst. Growth 180, $433-441$.

Davoust, L., Cowley, M. D., Moreau, R. \& Bolcato, R. 1999 Buoyancy-driven convection with a uniform magnetic field. Part 2. Experimental investigation. J. Fluid Mech. 400, 59-90.

Dold, P. \& BEnZ, K. W. 1995 Convective temperature fluctuations in liquid gallium in dependence on static and rotating magnetic fields. Cryst. Res. Technol. 30 (8), 1135-1145.

Dold, P. \& Benz, K. W. 1997 Modification of fluid flow and heat transport in vertical Bridgman configurations by rotating magnetic fields. Cryst. Res. Technol. 32 (1), 51-60. 
Garandet, J. P., Alboussière, T. \& Moreau, R. 1992 Buoyancy-driven convection in a rectangular enclosure with a transverse magnetic field. Intl J. Heat Mass Transfer 35 (4), 741-748.

Henry, D., Ben Hadid, H., Kaddeche, S. \& Dridi, W. 2008 a Magnetic stabilization of melt flows in horizontal Bridgman configurations. J. Cryst. Growth 310, 1533-1539.

Henry, D., Juel, A., Ben Hadid, H. \& Kaddeche, S. 2008b Directional effect of a magnetic field on oscillatory low-Prandtl-number convection. Phys. Fluids 20, 034104(1-12).

Hof, B., Juel, A. \& Mullin, T. 2003 Magnetohydrodynamic damping of convective flows in molten gallium. J. Fluid Mech. 482, 163-179.

Hof, B., Juel, A. \& Mullin, T. 2005 Magnetohydrodynamic damping of oscillations in low-Prandtlnumber convection. J. Fluid Mech. 545, 193-201.

Hurle, D. T. J. 1993 Crystal Pulling From the Melt. Springer-Verlag.

Hurle, D. T. J., Jakeman, E. \& Johnson, C. P. 1974 Convective temperature oscillations in molten gallium. J. Fluid Mech. 64, 565-576.

Juel, A., Mullin, T., Ben Hadid, H. \& Henry, D. 1999 Magnetohydrodynamic convection in molten gallium. J. Fluid Mech. 378, 97-118.

Kaddeche, S., Henry, D. \& Ben Hadid, H. 2003 Magnetic stabilization of the buoyant convection between infinite horizontal walls with a horizontal temperature gradient. J. Fluid Mech. 480, $185-216$.

Lyubimova, T. P., Lyubimov, D. V., Morozov, V. A., Scuridin, R. V., Ben Hadid, H. \& Henry, D. 2008 Stability of convection in a horizontal channel subjected to a longitudinal temperature gradient. Part 1. Effect of aspect ratio and Prandtl number. J. Fluid Mech. 635, 275-295.

MoreaU, R. 1990 Magnetohydrodynamics. Kluwer Academic.

OKADA, K. \& OzoE, H. 1992 Experimental heat transfer rates of natural convection of molten gallium suppressed under an external magnetic field in either the x-, y- or z-direction. ASME J. Heat Transfer 114, 107-114.

Ozoe, H. \& OKada, K. 1989 The effect of the direction of the external magnetic field on the three-dimensional natural convection in a cubical enclosure. Intl J. Heat Mass Transfer 32, 1939-1954.

Series, R. W. \& Hurle, D. T. J. 1991 The use of magnetic field in semiconductor crystal growth. J. Cryst. Growth 113, 305-327.

Utech, H. P. \& Flemings, M. C. 1966 Elimination of solute banding in Indium Antimonide crystals by growth in a magnetic field. J. Appl. Phys. 37 (5), 2021-2024. 